\title{
MULTISENSORY SIGNAL DETECTION: HOW DOES DRIVING AND IVIS MANAGEMENT AFFECT PERFORMANCE?
}

\author{
Natasha Merat and A. Hamish Jamson \\ Institute for Transport Studies \\ University of Leeds \\ Leeds, UK \\ E-mail: N.Merat@its.leeds.ac.uk
}

\begin{abstract}
Summary: Signal detection was used as a 'tertiary' task to assess drivers' 'spare processing capacity' during the performance of two in-vehicle information systems (IVIS). The main aims of the study were: a) to establish if performance on signal detection can be used to assess IVIS safety during driving and b) to determine whether signal modality is important for this assessment. Participants performed each IVIS (Phone or Count) during a driving simulation experiment. In addition to performing the driving and IVIS, participants were required to complete three detection tasks (DT): (i) a visual DT (ii) an auditory DT, and (iii) a tactile DT. Average reaction time to the DTs was found to increase by around $200 \mathrm{~ms}$ when performed with the IVIS tasks. It can be argued that any significant increase in reaction time to the DTs is a good indicator of drivers' reduced hazard perception/situation awareness, which might occur as a result of using in-car systems. No significant difference in performance was found between the various DTs, suggesting that performance relies on central attentional resources, and is not modality-specific. This affords some flexibility for assessing the safety of IVIS in different driving environments. For instance, an auditory DT might be used in field studies on a sunny day when bright light hinders detection of LEDs used in a visual version of the task. Similarly, the tactile version of the task might be useful for testing IVIS in a noisy driving environment.
\end{abstract}

\section{INTRODUCTION}

The function of most In-Vehicle Information Systems (IVIS) is to provide effective drivingrelated information and assistance. However, these systems can impose extra attentional demands on the driver, which, especially in situations of high driving-related workload, could be detrimental to road safety, as they remove drivers' attention away from the road ahead.

Recently, a large-scale field trial funded by the National Highway Traffic Safety Administration (the 100-car Naturalistic Study) found that driver inattention was associated with $78 \%$ of traffic collisions and $65 \%$ of near-collisions. Distractions by secondary tasks such as IVIS were found to be the main source of this inattention (Neale et al., 2005). Therefore, it is important that the effect of these secondary tasks on driving is evaluated in some way, to ensure that a particular IVIS does indeed provide information and assistance in a safe driving environment, rather than causing unnecessary distraction.

Previous studies have used the Peripheral Detection Task (PDT; Martens \& van Winsum, 1999) for such evaluations. Traditionally, this task involves measuring participants' reaction time to a 
series of LEDs, which are usually placed to the left of the driver's line of sight, on the dashboard of a car or driving simulator. Research has shown the PDT to be sensitive to variations in workload, such as that imposed by secondary tasks, or as a result of demanding road and traffic conditions (e.g., Olsson and Burns, 2000; van Winsum, et al.,1999).

The motivation for the current study came partly from earlier experiments, conducted for the European project HASTE (Human Machine Interaction and the Safety of Traffic in Europe), which revealed a concentration of gaze towards the centre of the road and a reduction in the frequency of drivers' peripheral saccades by a cognitively demanding non-visual 'surrogate' IVIS (Jamson and Merat, 2005; Victor, Harbluk and Engström, 2005). Therefore, one conclusion from these HASTE studies was that when performing a highly demanding non-visual IVIS, drivers' attention to peripheral visual events in the driving scene may be reduced, and this would clearly have detrimental effects on safety. To quantify the consequences of this reduction in peripheral saccades, the sensible next step was to examine the effect of such a demanding nonvisual IVIS on the Peripheral Detection Task. This was the first aim of the current study, as it was assumed that reduced detection of peripheral visual stimuli would signify the risks associated with using such IVIS during driving.

Clearly, using a visual detection task to study drivers' reduced attention towards the peripheral visual scene has its advantages, not least because visual signals are more ecologically valid. However, employing LEDs in the field has also been associated with visibility problems, brought about by the unpredictable changes in the background lighting of such natural settings (Recarte and Nunes, 2003; Engstrom, Åberg, Johansson, Hammerbäck, 2005). Therefore, there is some value in examining the suitability of other (non-visual) detection tasks for such assessments. The use of detection tasks in other modalities would also establish whether impaired signal detection in this context is specific to the visual modality or due to competition for central attentional resources. At present, two rather different bodies of literature can be used to predict how IVIS management in driving influences detection task performance across modalities.

On the one hand, some of the work on dual and multi-task performance in humans suggests that concurrent execution of two or more tasks is best accomplished when there is minimal overlap between processing stages, response codes and modalities for each task (e.g., Wickens and Holland, 2000). Therefore, from a multiple resources perspective, a visually presented IVIS is more detrimental to driving than a sound-based IVIS (e.g., Parkes and Coleman, 1990). This theory would also suggest that if drivers are required to respond to detection tasks in a 'triple task' condition involving driving, IVIS management and signal detection, impairment would be most prominent in a visual detection task, especially if a visual IVIS is employed. By the same token, better detection of tactile or auditory stimuli is expected in this type of triple-task driving condition. On the other hand, if a visually loading IVIS has the same effect on the detection of visual, tactile and auditory stimuli, it can be assumed that detection task performance is not modality specific, but relies on central attentional resources (see Strayer, Drew \& Johnston, 2003). If impaired signal detection is indeed due to competition for central attentional resources, the converse scenario is also likely, in that any demanding IVIS (whether visual or sound-based) will impair signal detection. Such a finding would provide some flexibility to researchers, allowing them to consider any one of the three types of detection task for the safety evaluation of IVIS in the field. For instance, visual and auditory versions of the task would be inappropriate to 
use in very sunny or noisy conditions, respectively, and IVIS safety can be evaluated with a tactile detection task instead.

To summarise, the aim of the current study was to evaluate the risks associated with using a nonvisual IVIS in driving, by measuring its effect on the detection of peripheral visual stimuli. To assess whether impairment is modality specific, performance in visual, auditory and tactile signal detection was compared. Additionally, to assess the effect of IVIS modality on detection across the three modalities, signal detection during management of a non-visual IVIS was compared to that of a visual IVIS.

Since a driving simulator was used in this study, the stimuli for the visual detection task were presented in the driving scene itself. This allowed us to create a task that was more analogous to detecting visual events in real-road conditions, since the detection task stimuli were seen at the same focal point as other objects in the scene. Broadband sounds and a vibrating tactor were used for the auditory and tactile detection tasks, respectively. The non-visual IVIS involved counting backwards in units of 7, and the visual IVIS required phone number entry on a touch screen monitor.

\section{METHOD}

\section{Participants}

The driving simulator database was used to recruit twenty-four drivers ( 8 male, 16 female) for this study. The mean age of drivers was 33 (range 19-58) and mean driving experience was 13 years (range 1-37). All drivers provided informed consent to participate in the experiments and received $£ 15$ upon completion of the study.

\section{Apparatus and materials}

The driving task. Driver behaviour was studied using the Leeds Advanced Driving Simulator (LADS). Data were collected at $60 \mathrm{~Hz}$.

The IVIS tasks. The visual IVIS or 'Phone' task, was displayed on a 6.4" touch screen LCD, placed to the left of the steering wheel (suitable for a UK-style right hand drive vehicle, Figure 1a). The 7-digit telephone numbers for this task were presented from a set of speakers positioned on the back parcel-shelf of the car. These speakers were also used to present the numbers for the non-visual 'Count' IVIS.

The detection tasks (DT). Presentation of all DTs was controlled by the simulator software. For the visual DT, a $.03 \mathrm{~m}$ red circle, subtending a visual angle of $0.68^{\circ}$ was presented randomly on the projection screen, to the left or right of the road scene, at a horizontal angle of $11-23^{\circ}$ and a vertical angle of $2-4^{\circ}$. The auditory DT consisted of a burst of broadband noise, presented through the car's speakers, which were located in the driver and passenger doors. The touch DT was presented via a small $1 \mathrm{~cm}^{2}$ vibrotactile pad (tactor) especially customised for the experiment. This was placed on the driver's neck, just below their left ear and fixed with 
micropore tape (Figure 1b). Response to all DTs was via a micro-switch attached to the right index finger (Figure 1c).

(a)

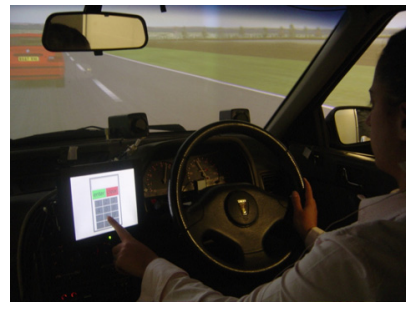

(b)

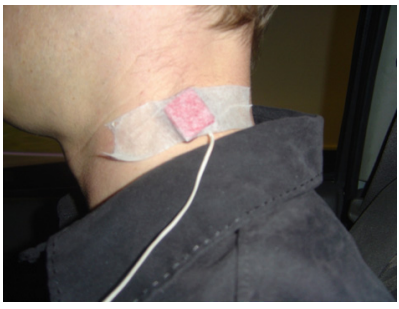

(c)

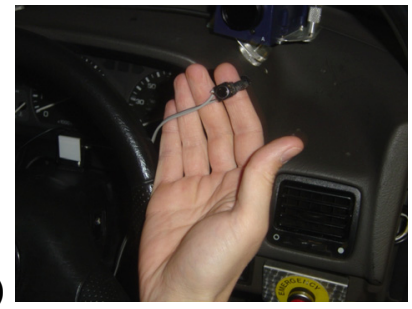

Figure 1. (a) The Phone task, (b) the tactor, (c) the microswitch used for all detection tasks

\section{Design and procedure}

All participants were provided with written instructions for the experiment before completing the consent form. After practicing each IVIS and DT in isolation, they practiced the driving session, which involved a car-following task in a rural environment. Participants were asked not to overtake the lead car, but follow it as they would in a real driving situation. They had the opportunity to practice the driving task in isolation, and were also given the chance to practice driving with the IVIS and DTs.

A within-subjects design was used with all drivers completing three runs of driving: one run with only the DTs (i.e., No IVIS) and two runs with the DTs plus either the Phone or Count IVIS. Baseline (single task) driving was also included in the 'No IVIS' condition. This created three conditions within the runs: (i) single task condition: baseline driving (ii) dual task condition: driving with one of the three DTs or driving

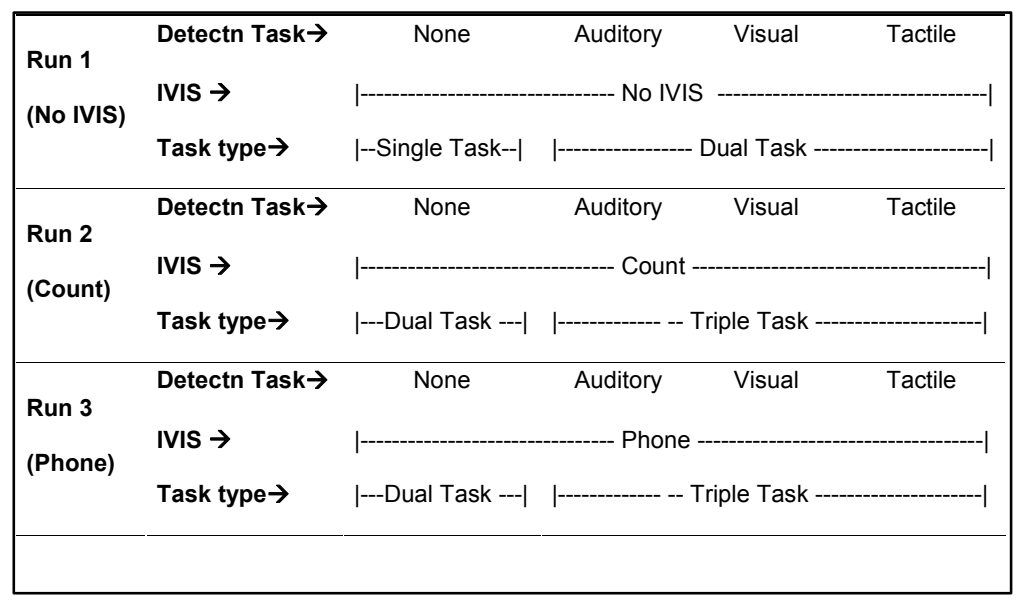

Figure 2. An example of the experimental design with one of the IVIS tasks, and (iii) triple task condition: driving with one of the three DTs and one of the two IVIS (Figure 2). The order of the three driving runs was counterbalanced across participants. The DTs were presented in 60-second blocks during all three runs, with each block of DT presented randomly within a particular experimental run. This ensured that driving without a DT was not always conducted at the start of a run. During the triple task conditions, the start of each DT coincided with the start of an IVIS task. No explicit instructions were provided regarding prioritisation of the IVIS and DTs, but drivers were reminded that driving was their primary task.

For the Phone IVIS task, drivers heard a series of 7-digit telephone numbers, which were prerecorded and played at predetermined sections of the road, using speakers on the parcel-shelf of 
the car. Drivers were asked to enter each block of 7-digits as quickly and accurately as possible on the touch screen monitor, followed by the 'enter' key. The next 7-digit telephone number was then presented after the 'enter' key was pressed. This Phone task lasted 60 seconds, and the speed and accuracy of drivers' input was recorded. For the Count IVIS task, drivers heard a prerecorded three digit number, which was presented from the car's parcel-shelf speakers. They were asked to count aloud backwards from this number, in units of 7, and were then told to stop counting after 60 seconds had lapsed. Drivers' response to this task was recorded by the experimenter. The stimuli for the detection tasks were presented at a random rate of once every 2 to 5 seconds and each stimulus remained activated for 2 seconds, or until the driver pressed the response button.

\section{RESULTS}

Participants' performance in the driving task, IVIS tasks and detection tasks was examined for all of the task combinations shown in Figure 2. However, due to limitations of space, only performance on the detection tasks is reported here. The dependent variables for the detection tasks were hit rate and response time (RT). In line with previous studies (e.g., Patten et al., 2003) 'hits' were defined as stimuli with response times between $200 \mathrm{~ms}$ and 2 seconds. Hit rate was then defined as the number of hits divided by the total number of stimuli. Average response time for these hits was also computed. All missed responses (which included anything above 2 seconds and all 'no' responses) and guesses (anything between 0 and $200 \mathrm{~ms}$ ) were also considered during data analysis, although the results of these are not reported here.

The effect of IVIS management on DT performance was assessed by comparing average RT in dual task performance (driving + DT) with triple task performance (driving + DT + IVIS). Since response to each detection task stimulus is related to the relative ease with which signals are localized in that modality, as well as the transduction latency of each of the senses (e.g., see Spence and Squire, 2003) separate analyses of variance were conducted for each DT, using a 3 factor (No IVIS, Count, Phone) repeated measures ANOVA. Results showed a significant rise in reaction time for all detection tasks with the addition of the Count and Phone IVIS. As shown in Table 1, post hoc Bonferroni tests also showed significantly higher response times as a result of the Phone IVIS, compared to the Count IVIS.

Table 1. Reaction time to each detection task during driving and 'driving + IVIS'

\begin{tabular}{l|lllll} 
Detection task & Driving only & Driving plus IVIS & df & F & Significance \\
\hline Auditory & 442.96 & $562.22^{*}(\mathrm{C}), 779.32^{* \dagger}(\mathrm{P})$ & 2,46 & 58.26 & $\mathrm{p}<.001$ \\
Visual & 472.40 & $622.21^{*}(\mathrm{C}), 826.58^{* \dagger}(\mathrm{P})$ & 2,46 & 130.76 & $\mathrm{p}<.001$ \\
\hline Tactile & 405.17 & $523.66^{*}(\mathrm{C}), 669.79^{\dagger}(\mathrm{P})$ & 2,46 & 43.47 & $\mathrm{p}<.001$
\end{tabular}

*Significanlty different from driving only condition $(\mathrm{p}<.05) . \mathrm{C}=\mathrm{Count}, \mathrm{P}=\mathrm{Phone}$

${ }^{\dagger}$ Significantly different from Count $(\mathrm{p}<.05)$.

To compare the effect of each IVIS across the three DTs, and because transduction latency is different for each modality, RT values were standardized by dividing the triple task RT for each 
detection task by its corresponding dual task value. A repeated measures ANOVA with withinsubject factors IVIS (Phone, Count) and DT (visual, auditory, tactile) was then conducted on these standardized values. Results showed a significant effect of IVIS $(F(1,23)=120.01, p<$ $.001)$ with higher reaction times as a result of the Phone task. However, for each of the two IVIS conditions, there was no difference in RT across the three detection tasks (see Figure 3).

To examine the effect of each IVIS on hit rates, a similar calculation was conducted, by expressing triple task hit rate as a proportion of its dual task equivalent. Repeated measures ANOVA with within-subjects factors IVIS (Phone, Count) and DT (auditory, visual, tactile) showed a significantly higher hit rate during the Count task $(\mathrm{F}(1$, $23)=14.01, p<.05)$. However, there was no main effect of detection task and no interaction between IVIS and detection task.

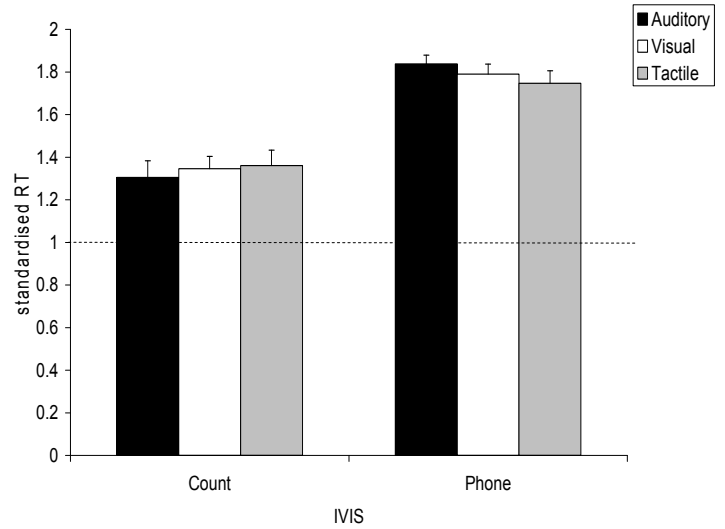

Figure 3. The effect of each IVIS on standardized RT for the 3 DTs

\section{DISCUSSION AND CONCLUSIONS}

This study examined the effect of two in-vehicle information systems on signal detection during driving. Detection of visual signals was assumed to be analogous to drivers' ability to respond to sudden and unexpected events in the road. Therefore, any impairment in visual signal detection as a result of IVIS management was thought to highlight the risks associated with using a demanding IVIS during driving, and a suitable method for evaluating the safety of IVIS. To investigate whether performance in signal detection is modality specific, the effect of performing a visual and non-visual IVIS on signal detection in the visual, auditory and tactile modality was also examined.

Average response time to the detection tasks was found to increase from the dual task condition $(\mathrm{DT}+$ driving) to the triple task condition (DT + driving + IVIS). This increase in response time was also significantly different between the two IVIS tasks, with higher reaction times seen during management of the Phone task. This higher reaction time associated with the Phone task might be due to a conflict between the manual response required for both the Phone IVIS and the detection tasks. Therefore, future studies would benefit from examining the effect of such performance in signal detection when a verbal response is used for the visual IVIS task.

The impairment caused by the Phone and Count IVIS was the same across all three detection tasks. This absence of a difference in performance as a function of modality is important, as it suggests that the effect of IVIS on detection is not modality specific but relies on central attentional resources. Therefore, performing a demanding IVIS can reduce drivers' ability to respond to stimulus-driven events, and our results suggest that this degradation is not modality specific, but relies on central attentional resources. 
In conclusion, the results of the current study indicate that signal detection tasks similar to those used here are suitable for quantifying the safety of IVIS, as reduced signal detection during IVIS management can be directly associated with reduced attention towards unexpected events in the road. An absence of a difference in detection task performance across the three modalities also affords some flexibility in this evaluation, allowing the use of the most suitable detection task for the particular experimental setup.

\section{ACKNOWLEDGMENTS}

The authors would like to thank Anthony Horrobin for creating the simulator scenarios. This study was funded by the European project AIDE IST-1-507674-IP.

\section{REFERENCES}

Jamson, A.H., \& Merat, N. (2005). Surrogate In Vehicle Information Systems and Behaviour: Effects of Visual and Cognitive Load in Simulated Driving. Transportation Research Part F: Traffic Psychology and Behaviour, 8(2), 79-96.

Martens, M., \& van Winsum, W. (1999). Activation criteria and warning strategies: Driving simulator results. IN-ARTE Project (TR4014), ID 7.1. Unpublished technical report. TNO Soesterberg.

Neale, V.L., Dingus, T.A., Klauer, S.G., Sudweeks, J., \& Goodman, M. (2005). An overview of the 100-car naturalistic study and findings. National Highway Traffic Safety Administration. Available at: http://www-nrd.nhtsa.dot.gov/pdf/nrd-12/100Car_ESV05summary.pdf

Olsson, S., \& Burns, P.C. (2000). Measuring driver visual distraction with a Peripheral Detection Task. Linköping, Sweden: Linköping University.

Parkes, A.M., \& Coleman, N. (1990). Route guidance systems: a comparison of methods of presenting directional information to the driver. In E.J. Lovesey (ed.) Contemporary Ergonomics. London: Taylor \& Francis, 480-485.

Recarte, M., \& Nunes, L. (2003). Mental Workload While Driving: Effects on Visual Search, Discrimination, and Decision Making. Journal of Experimental Psychology: Applied. 9(2), 119-137.

Strayer, D.L., Drews, F.A. \& Johnston, W.A. (2003). Cell Phone-Induced Failures of Visual Attention During Simulated Driving, Journal of Experimental Psychology: Applied. 9(1), 23-32.

van Winsum, W., Martens, M. \& Herland, L. (1999). The effects of speech versus tactile driver support messages on workload, driver behaviour and user acceptance. Report TNO Human Factors Research Institute, Soesterberg, the Netherlands.

Wickens, C.D., \& Hollands, J.G. (2000). Complex systems, process control, and automation. In C.D. Wickens \& J.G. Hollands (Eds.), Engineering psychology and human performance ( $3^{\text {rd }}$ ed.). Upper Saddle River, NJ: Prentice Hall, 538-550. 